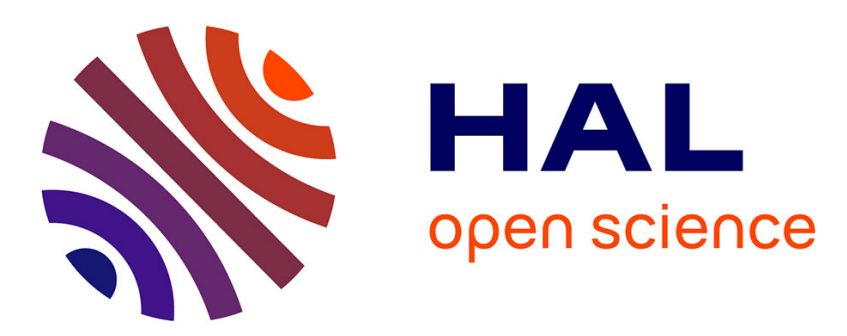

\title{
The random map model: a disordered model with deterministic dynamics
}

\author{
B. Derrida, H. Flyvbjerg
}

\section{To cite this version:}

B. Derrida, H. Flyvbjerg. The random map model: a disordered model with deterministic dynamics. Journal de Physique, 1987, 48 (6), pp.971-978. 10.1051/jphys:01987004806097100 . jpa-00210526

\section{HAL Id: jpa-00210526 https://hal.science/jpa-00210526}

Submitted on 1 Jan 1987

HAL is a multi-disciplinary open access archive for the deposit and dissemination of scientific research documents, whether they are published or not. The documents may come from teaching and research institutions in France or abroad, or from public or private research centers.
L'archive ouverte pluridisciplinaire HAL, est destinée au dépôt et à la diffusion de documents scientifiques de niveau recherche, publiés ou non, émanant des établissements d'enseignement et de recherche français ou étrangers, des laboratoires publics ou privés. 


\title{
The random map model : a disordered model with deterministic dynamics
}

\author{
B. Derrida $\left({ }^{*}\right)$ and H. Flyvbjerg $\left(^{+}\right)$ \\ Institute for Theoretical Physics, University of California Santa Barbara, CA 93106, U.S.A. \\ $\left(^{+}\right)$The Nields Bohr Institute, University of Copenhagen Blegdamsvej 17, DK-2100 Copenhagen $\varnothing$, \\ Denmark
}

(Reçu le 17 novembre 1986, accepté le 9 février 1987)

Résumé. - Le modèle d'application aléatoire que nous considérons est un modèle désordonné simple dont la
dynamique est aléatoire. Pour chaque point de l'espace des phases, on choisit au hasard un autre point de
l'espace des phases comme étant son successeur. L'espace des phases se décompose en plusieurs bassins
d'attraction. Nous obtenons l'expression analytique de la distribution $f\left(W_{s}\right)$ des poids $W_{s}$ où $W_{s}$ représente la
taille normalisée du bassin du $s$-ième attracteur. Nous calculons aussi la distribution $\pi(Y)$ de $Y$ ou
$Y=\sum_{s} W_{s}^{2}$. Quand on compare $f(W)$ et $\pi(Y)$ du modèle du mapping aléatoire avec ce qui a été obtenu dans la
théorie du champ moyen des verres de spins, on trouve que, dans les deux problèmes, ces lois des probabilité ont des formes très semblables mais des expressions différentes.

\begin{abstract}
The random map model is a simple disordered system with deterministic dynamics. For each point in phase space, one chooses at random another point in phase space as being its successor in time. Phase space is broken into basins of several attractors. We obtain the analytic expression for the probability distribution $f\left(W_{s}\right)$ of the weights $W_{s}$, where $W_{s}$ denotes the normalized size of the basin of the $s$-th attractor. We also compute the probability distribution $\pi(Y)$ of $Y$ where $Y$ is defined by $Y=\sum_{s} W_{s}^{2}$. When we compare $f(W)$ and $\pi(Y)$ in the random map model and in the mean field theory of spin glasses, we find that the shapes are very similar in both models but the analytic expressions are different.
\end{abstract}

\section{Introduction.}

The purpose of the present work is to give an analytic solution of the random map model which is a limiting case of the Kauffman model. We will compute the statistical properties of the multivalley structure. Our main conclusion will be that the random map model has not the same multivalley structure as the Sherington-Kirkpatrick model. The expressions for the probability distributions of the sizes of valleys and their shapes are nevertheless very similar in the two models.

In the present work, we shall associate to each

(*) Permanent address : SPT, CEN, Saclay, F-91191 Gif-sur-Yvette, France. attractor $s$ a weight $W_{s}$ which is the normalized number of points which fall in the $s$-th basin of attraction. So if phase space consists of $M$ points and $\Omega_{s}$ of these points fall on the $s$-th attractor, we have

$$
W_{s}=\Omega_{s} / M \text {. }
$$

We compute $f(W)$ which is defined in the following way : $f(W) \mathrm{d} W$ is the average number of attractors with weight between $W$ and $W+\mathrm{d} W$. We also compute the probability distribution $\pi(Y)$ of $Y$ where $Y$ is defined by

$$
Y=\sum_{s} W_{s}^{2}
$$

The paper is organized as follows : in section 2, we define the random map model, and give a brief 
summary of already known results of the random model, in particular why it is a limiting case of the Kauffman model. In section 3, we compute the moments $\overline{W^{n}}$ in the thermodynamical limit and we obtain $f(W)$. We also give the expression for the probability $g\left(W_{1}, W_{2}\right)$ that two randomly chosen configurations fall on attractors of weight $W_{1}$ and $W_{2}$. Lastly we obtain the probability $h(W, l)$ that a randomly chosen initial configuration falls on an attractor of weight $W$ and of period $l$. In section 4, we obtain a simple recursion relation for the moments $\overline{Y^{n}}$ and we draw $\pi(Y)$. We also give the shape of the probability distribution of the weight of the largest basin of attraction.

\section{Definition of the random map model.}

One considers a system whose phase space consists of $M$ points. The dynamics of the system is governed by a random map of this set of $M$ points into itself : for each point $1 \leqq i \leqq M$, one chooses at random one point $T(i)$ among the $M$ points of phase space as being the successor in time of point $i$. So if the system is in configuration (or point) $i$ at time $t$, it will be on point $T(i)$ at time $t+1$. If it comes back at a later time $t^{\prime}$ to configuration $i$, it will visit again configuration $T(i)$ at time $t^{\prime}+1$.

This model is random because the map $T$ is random. So, at least for finite $M$, the properties of this system should depend on the choice of the map $T$ and are therefore not selfaveraging. On the other hand, the dynamics are deterministic because $T$ does not change with time. This implies that the time evolution of any initial configuration ends up being periodic (just because phase space is finite and after a time $t>M$, the system must have visited twice the same configuration). So the set of $M$ points is the union of the basins of several periodic attractors.

In the present work, we will compute the properties of this random map model in the thermodynamic limit $(M \rightarrow \infty)$. We will see that in this limit, several properties remain non-selfaveraging, in a way very similar to what happens in the mean field theory of spin glasses [17-20].

The statistical properties of a random map of a set of $M$ points into itself have been studied a long time ago in the theory of probability [21-25] and has more recently attracted the attention of physicists $[26,27]$. Reference [21] gives a review of results known on the subject. Let us first mention some of these results which are related to the calculations presented in the next sections of this paper or which we found particularly interesting.

The probability $P(l)$ that a randomly chosen initial configuration ends up on an attractor of period $l$ is

$$
P(l)=\sum_{n \geqq l} \frac{\Gamma(M)}{\Gamma(M-n+1)} \frac{1}{M^{n}} .
$$

In the limit $M \rightarrow \infty$, one gets

$$
P(l)=\frac{1}{\sqrt{M}} \int_{x}^{\infty} \mathrm{d} y \mathrm{e}^{-y^{2} / 2}
$$

where $x$ is defined by

$$
l=\sqrt{M} x .
$$

This gives for the average $\langle l\rangle$ and for the variance

$$
\langle l\rangle=\sqrt{M} \sqrt{\frac{\pi}{8}} ;\left\langle l^{2}\right\rangle-\langle l\rangle^{2}=M\left(\frac{2}{3}-\frac{\pi}{8}\right) \text {. }
$$

The probability $Q_{M}$ that a random map of $M$ points is indecomposable $[24,25]$ is given by :

$$
Q_{M}=\frac{(M-1) !}{M^{M}} \sum_{n=0}^{M-1} \frac{M^{n}}{n !}
$$

Indecomposability means that the map has a single attractor. So with probability $Q_{M}$, the $M$ points fall on the same attractor. For large $M$ this gives

$$
Q_{M} \sim \sqrt{\frac{\pi}{2 M}} .
$$

Reference [24] gives also the probability $Q_{M}(l)$ that the map is indecomposable and that the attractor has a period $l$ :

$$
Q_{M}(l)=\frac{M !}{(M-l) ! M^{l+1}} .
$$

Another interesting result concerns the probability distribution that a random map has exactly $A$ attractors. The probability distribution of $A$ is known [21]. Let us just give here the result [22, 27] for the average number $\langle A\rangle$ of attractors of a random map of $M$ points :

$$
\langle A\rangle=\frac{1}{2} \log M+\mathcal{O}(1) .
$$

Reference [21] gives several other interesting results which we will not mention here. In particular how the previous results can be generalized to random maps with constraints : for example such that $T(i) \neq i$, or one to one maps.

Let us now see why the random map model is a limiting case of the Kauffman model. In the Kauffman model, one considers a system of $N$ sites, each site containing an Ising spin $\left(\sigma_{i}=1\right.$ or 0$)$. For each site $i$, one chooses at random $K$ input sites (among the $N$ sites) $j_{1}(i), j_{2}(i), \ldots, j_{K}(i)$ and a random Boolean function $f_{i}$ of $K$ variables. The time evolution of the system is given by

$\sigma_{i}(t+1)=f_{i}\left(\sigma_{j_{1}(i)}(t), \sigma_{j_{2}(i)}(t), \ldots, \sigma_{j_{K}(i)}(t)\right)$.

Notice that since each of the $K$ input sites 
$j_{1}(i), \ldots, j_{K}(i)$ is chosen at random and independently of preceeding choices, the $K$ input sites are not necessarily different. Therefore $K$ needs not be less than $N$.

If one considers two configurations of spins $\mathrm{C}_{1}$ and $\mathrm{C}_{2}$ which are at distance $n$ at time $t=0$, one can compute the probability $P_{1}(m, n)$ that their distance is $m$ at time $t=1$. (Here distance means the number of spins which are different in the two configurations.) The expression of $P_{1}(m, n)$ was derived in reference [6] :

$$
\begin{aligned}
& P_{1}(m, n)=\frac{N !}{m !(N-m) !} \frac{1}{2^{N}} \times \\
& \quad \times\left(1+\left(1-\frac{n}{N}\right)^{K}\right)^{N-m}\left(1-\left(1-\frac{n}{N}\right)^{K}\right)^{m} .
\end{aligned}
$$

In the limit $K \rightarrow \infty$ (which can be taken even for finite $N$ since, one finds

$\begin{array}{lll}P_{1}(m, n)=\frac{N !}{m !(N-m) !} \frac{1}{2^{N}} & \text { for } & n \neq 0 \\ P_{1}(m, n)=\delta_{m, 0} & \text { for } & n=0 .\end{array}$

This shows that in the limit $K \rightarrow \infty$, the images by the dynamics of two configurations in phase space are not correlated even if the two configurations $\mathrm{C}_{1}$ and $\mathrm{C}_{2}$ are very close. This limit $K \rightarrow \infty$ of the Kauffman model is very reminiscent of the limit $p \rightarrow \infty$ of the $p$ spin glass model which gives the Random Energy model where the energies become incorrelated [28].

\section{Distribution of the sizes of valleys.}

In order to compute the probability distribution of the weights $W$, we are going to compute the probability $\bar{Y}_{n}$ that $n$ randomly chosen initial configurations fall on the same attractor. Let us define $Q\left(T_{1}, T_{2}, \ldots, T_{n}\right)$ by

$$
\begin{aligned}
Q\left(T_{1}, T_{2}, \ldots, T_{n}\right)= \\
=\left(1-\frac{1}{M}\right)\left(1-\frac{2}{M}\right) \\
\quad \ldots\left(1-\frac{T_{1}+T_{2}+\cdots+T_{n}-1}{M}\right) \\
\quad \times \frac{T_{1}^{2}}{M^{2}} \cdot \frac{T_{1}+T_{2}}{M} \cdots \frac{T_{1}+T_{2}+\cdots+T_{n-1}}{M}
\end{aligned}
$$

$\left(Q\left(T_{1}, T_{2}, \ldots, T_{n}\right)\right.$ is the probability that a randomly chosen configuration $\mathrm{C}_{1}$ at time $t=0$ visits $T_{1}$ different points of phase space (at times $\left.t=0,1, \ldots, T_{1}-1\right)$ ) before it falls on a point already visited, that a randomly chosen configuration $\mathrm{C}_{2}$ visits $T_{2}$ different points of phase space before it falls on the trajectory of $\mathrm{C}_{1}, \ldots$ that a randomly chosen configuration $\mathrm{C}_{n}$ visits exactly $T_{n}$ different points before it falls on the union of the trajectories of $\mathrm{C}_{1}, \mathrm{C}_{2}, \ldots, \mathrm{C}_{n-1}$.

Then the probability $\bar{Y}_{n}$ that the $n$ configurations fall on the same attractor is just

$\bar{Y}_{n}=\sum_{T_{1}=1}^{\infty} \sum_{T_{2}=0}^{\infty} \sum_{T_{3}=0}^{\infty} \cdots \sum_{T_{n}=0}^{\infty} Q\left(T_{1}, T_{2}, \ldots, T_{n}\right)$.

If one makes the change of variable

$$
S_{i}=\sum_{j=1}^{i} T_{j}
$$

Then (13) and (14) become

$$
\begin{gathered}
\bar{Y}_{n}=\sum_{s_{n}=1}^{\infty} \sum_{S_{n-1}=1}^{s_{n}} \cdots \sum_{s_{1}=1}^{s_{2}} \frac{S_{1}^{2}}{M^{2}} \frac{S_{2}}{M} \frac{S_{3}}{M} \cdots \\
\cdots \frac{S_{m-1}}{M}\left(1-\frac{1}{M}\right)\left(1-\frac{2}{M}\right) \\
\cdots\left(1-\frac{S_{n}-1}{M}\right) .
\end{gathered}
$$

In the limit $M \rightarrow \infty$, one can use continuous variables :

$$
S_{i}=\sqrt{M} t_{i} ; \quad 1=\sqrt{M} \mathrm{~d} t_{i}
$$

and (16) becomes

$$
\begin{aligned}
\bar{Y}_{n}=\int_{0}^{\infty} \mathrm{d} t_{n} \mathrm{e}^{-t_{n}^{2} / 2} \int_{0}^{t_{n}} \mathrm{~d} t_{n-1} t_{n-1} \times \\
\times \int_{0}^{t_{n-1}} \mathrm{~d} t_{n-2} t_{n-2} \cdots \int_{0}^{t_{2}} \mathrm{~d} t_{1} t_{1}^{2}
\end{aligned}
$$

and one finds

$$
\begin{aligned}
& \bar{Y}_{n}=\frac{4^{n-1}[(n-1) !]^{2}}{(2 n-1) !}= \\
& =\frac{1}{2} \cdot \frac{\Gamma(n) \Gamma\left(\frac{1}{2}\right)}{\Gamma\left(n+\frac{1}{2}\right)}=\frac{1}{2} B\left(n, \frac{1}{2}\right)
\end{aligned}
$$

where $B(y, z)$ is Euler's integral of the first kind $\left(\bar{Y}_{2}=2 / 3 ; \bar{Y}_{3}=8 / 15 ; \bar{Y}_{4}=16 / 35 ; \bar{Y}_{5}=128 / 315\right.$ etc.). For a given sample (i.e. for a given random map) the probability $Y_{n}$ that $n$ configurations fall on the same attractor is

$$
Y_{n}=\sum_{s} W_{s}^{n}
$$

Therefore, when one averages over the disorder, one has

$$
\bar{Y}_{n}=\int_{0}^{1} \mathrm{~d} W f(W) W^{n}
$$

where $f(W) \mathrm{d} W$ is the average number of attractors which have a weight between $W$ and $W+d W$. 
Using the well known relation

$$
\begin{aligned}
B(y, z)=\int_{0}^{1} \mathrm{~d} W W^{y-1}(1-W)^{z-1} & = \\
& =\frac{\Gamma(y) \Gamma(z)}{\Gamma(y+z)}
\end{aligned}
$$

one gets from (19) and (21):

$$
f(W)=\frac{1}{2} W^{-1}(1-W)^{-1 / 2}
$$

If one wants the probability $g(W)$ that a randomly chosen configuration belongs to a basin of attraction of weight $W$, it is just

$$
g(W)=f(W) W=\frac{1}{2}(1-W)^{-1 / 2}
$$

$g(W)$ is the probability $f(W)$ of having an attractor of weight $W$ times the probability $W$ of falling on this attractor. .

Knowing $f(W)$, it is easy to compute $g\left(W_{1}, W_{2}\right)$, the probability that two randomly chosen configurations $\mathrm{C}_{1}$ and $\mathrm{C}_{2}$ fall on attractors of weight $W_{1}$ and $W_{2}$ respectively :

$$
\begin{aligned}
& g\left(W_{1}, W_{2}\right) \mathrm{d} W_{1} \mathrm{~d} W_{2}=g\left(W_{1}\right) W_{1} \delta\left(W_{1}-W_{2}\right) \times \\
& \quad \times \mathrm{d} W_{1} \mathrm{~d} W_{2}+g\left(W_{1}\right)\left(1-W_{1}\right) g\left(\frac{W_{2}}{1-W_{1}}\right) \\
& \quad \times \mathrm{d} W_{1} \mathrm{~d}\left(\frac{W_{2}}{1-W_{1}}\right) .
\end{aligned}
$$

In (25) the first term is the probability that $\mathrm{C}_{1}$ and $\mathrm{C}_{2}$ fall on the same attractor of weight $W_{1}$. The second term expresses the following: if $\mathrm{C}_{1}$ falls on an attractor of weight $W_{1}$, this means that phase space can be decomposed into $M W_{1}$ points which fall on the same attractor as $\mathrm{C}_{1}$ and $M\left(1-W_{1}\right)$ other points. The restriction of the random map $T$ to these $M\left(1-W_{1}\right)$ points is again a random map $T$ of $M\left(1-W_{1}\right)$ points into themselves.

The probability of finding an attractor of weight $W$ for this restricted map $T$ is again given by $f(W)$. An attractor having a weight $W$ for the restricted map $T$ has of course a weight $\left(1-W_{1}\right) W$ for the full map $T$. From (24) and (25) one gets

$$
\begin{aligned}
g\left(W_{1}, W_{2}\right)=\frac{1}{2} W_{1}(1 & \left.-W_{1}\right)^{-1 / 2} \delta\left(W_{1}-W_{2}\right)+ \\
& +\frac{1}{4}\left(1-W_{1}-W_{2}\right)^{-1 / 2}
\end{aligned}
$$

Similarly one can calculate $g\left(W_{1}, W_{2}, W_{3}\right)$, the probability that if $\mathrm{C}_{1}, \mathrm{C}_{2}$, and $\mathrm{C}_{3}$ are randomly chosen, they fall on attractors of weights $W_{1}, W_{2}$ and $W_{3}$ :

$$
\begin{aligned}
& g\left(W_{1}, W_{2}, W_{3}\right)= \\
& =\frac{1}{2}\left(1-W_{1}\right)^{-1 / 2} \delta\left(W_{1}-W_{2}\right) \delta\left(W_{1}-W_{3}\right) W_{1}^{2}
\end{aligned}
$$

$$
\begin{aligned}
& +\frac{1}{4}\left[\left(1-W_{1}-W_{2}\right)^{-1 / 2} \delta\left(W_{1}-W_{3}\right) W_{1}\right. \\
& +\left(1-W_{1}-W_{2}\right)^{-1 / 2} \delta\left(W_{2}-W_{3}\right) W_{2} \\
& \left.+\left(1-W_{1}-W_{3}\right)^{-1 / 2} \delta\left(W_{1}-W_{2}\right) W_{1}\right] \\
& +\frac{1}{8}\left(1-W_{1}-W_{2}-W_{3}\right)^{-1 / 2} .
\end{aligned}
$$

We can now compare the results (23) and (26) with what is known in the mean field theory of spin glasses [18]. Like in the case of spin glasses, the average number of valleys is infinite (it is given by $\int_{0}^{1} f(W) \mathrm{d} W$ which diverges). This agrees of course with equation (9) which implies that for $M \rightarrow \infty$, the average number of valleys is infinite.

In spin glasses, the expression of $f(W)$ and of $f\left(W_{1}, W_{2}\right)=g\left(W_{1}, W_{2}\right) /\left(W_{1}, W_{2}\right)$ are [18] :

$$
f_{\mathrm{SG}}(W)=\frac{W^{y-2}(1-W)^{-y}}{\Gamma(y) \Gamma(1-y)}
$$

$$
\begin{aligned}
& f_{\mathrm{SG}}\left(W_{1}, W_{2}\right)=\delta\left(W_{1}-W_{2}\right) f_{\mathrm{SG}}\left(W_{1}\right)+ \\
& +(1-y) \frac{\left(W_{1} W_{2}\right)^{y-2}\left(1-W_{1}-W_{2}\right)^{1-2 y}}{\Gamma(y) \Gamma(y) \Gamma(2-2 y)}
\end{aligned}
$$

where $y$ is a parameter which depends on the model, the temperature, the magnetic field etc., etc. We see that the expressions (28) and (29) are surprisingly similar to (23) and (26) although there is no choice of $y$ for which these expressions would be identical so the multivalley structure of the random map is qualitatively very similar to what is known in the mean field theory of spin glasses but the two problems are nevertheless different.

One can wonder whether the weight $W$ of an attractor is correlated to the period $l$ of this attractor. It is possible to calculate the probability $h(W, l)$ that a randomly chosen configuration falls on an attractor of weight between $W$ and $W+\mathrm{d} W$ and of period $l$. One can follow the same steps as at the beginning of this section. If $\overline{Z_{n}(l)}$ is the probability that $n$ randomly chosen configurations fall on an attractor of period $l$, one has

$$
\begin{aligned}
& \overline{Z_{n}(l)}=\sum_{s_{n}=l}^{\infty} \sum_{S_{n-1}=l}^{s_{n}} \cdots \sum_{s_{1}=l}^{s_{2}} \frac{1}{M} \frac{S_{1}}{M} \frac{S_{2}}{M} \cdots \\
& \ldots \frac{S_{n-1}}{M}\left(1-\frac{1}{M}\right)\left(1-\frac{2}{M}\right) \\
& \cdots\left(1-\frac{S_{n}-1}{M}\right) \text {. }
\end{aligned}
$$

The only difference with formula (16) is that the sums start for $S_{i}=l$ and that the term $S_{1}^{2} / M^{2}$ has been replaced by $S_{1} / M^{2}$. One can again use continuous variables for $M \rightarrow \infty$, and one gets

$$
\begin{aligned}
\overline{Z_{n}(l)}=\frac{1}{\sqrt{M}} \frac{1}{(n-1) !} \frac{1}{2^{n-1}} \times \\
\quad \times \int_{x}^{\infty} \mathrm{d} y \mathrm{e}^{-y^{2} / 2}\left(y^{2}-x^{2}\right)^{n-1}
\end{aligned}
$$


where $x$ is defined by

$$
l=\sqrt{M} x .
$$

After some manipulations (see the appendix), one can show that

$$
\overline{Z_{n}(l)}=\int_{0}^{1} \mathrm{~d} W W^{n-1} h(W, l)
$$

where

$$
\begin{aligned}
& h(W, l)=h(W, \sqrt{M} x)= \\
& \quad=\frac{1}{\sqrt{2 \pi M}} \frac{1}{\sqrt{W(1-W)}} \mathrm{e}^{-\frac{x^{2}}{2 W}} .
\end{aligned}
$$

Of course, if one sums over $l$, one recovers $g(W)$ given by $(24)$

$$
g(W)=\sum_{l} h(W, l)=\sqrt{M} \int_{0}^{\infty} \mathrm{d} x h(W, \sqrt{M} x)
$$

and if one sums over $W$, one recovers $P(l)$ given by (4) :

$$
P(l)=\int_{0}^{1} \mathrm{~d} W h(W, l)=\overline{Z_{1}(l)} .
$$

Expression (34) shows how the weight of an attractor is correlated to its period. The main effect is that if the period $l$ is large $(x=l / M$ large $)$, then the weight $W$ cannot be small.

To conclude this section we would like to show how (24) can be derived also from (7).

Let us call $R_{n}$ the probability that configuration $\mathrm{C}_{1}$ falls on an attractor which has $n$ points in its basin. One has

$$
\begin{aligned}
R_{n}=\frac{(M-1) !}{(n-1) !(M-n) !} \times \\
\quad \times\left(1-\frac{n}{M}\right)^{M-n}\left(\frac{n}{M}\right)^{n} Q_{n}
\end{aligned}
$$

which is found the following way:

$$
(M-1) ! /(n-1) ! /(M-n) !
$$

is the number of ways one can choose $n$ configurations with configuration $\mathrm{C}_{1}$ among them. $(n / M)^{n} Q_{n}$ is the probability that these $n$ configurations map into themselves (see Eq. (7)). Finally $(1-n / M)^{M-n}$ is the probability that the remaining $M-n$ configurations do not map into the set of $n$ configurations which constitute the basin of attraction of $\mathrm{C}_{1}$. In the limit $M \rightarrow \infty$, if one writes

one gets

$$
n=W M
$$

$$
R_{n}=\frac{1}{2 M} \frac{1}{\sqrt{1-W}}
$$

So the probability that a configuration falls on an attractor of weight between $W$ and $W+\mathrm{d} W$ is

$$
g(W) \mathrm{d} W=R_{n} M \mathrm{~d} W .
$$

Very similar calculations can be done to derive (26) and (27) from (7).

One can also easily recover (34). Exactly as (37) was obtained, one can compute the probability $R_{n}(l)$ that configuration $\mathrm{C}_{1}$ falls on an attractor which has $n$ points in its basin and a period $l$. One has

$$
\begin{aligned}
& R_{n}(l)=\frac{(M-1) !}{(n-1) !(M-n) !} \times \\
& \quad \times\left(1-\frac{n}{M}\right)^{M-n}\left(\frac{n}{M}\right)^{n} Q_{n}(l)
\end{aligned}
$$

where $Q_{n}(l)$ was given in equation (8). For $M$ and $l$ large, if we set

$$
\begin{aligned}
n & =M W \\
l & =\sqrt{M} x
\end{aligned}
$$

we get

$$
R_{n}(l)=\frac{1}{\sqrt{2 \pi W(1-W)}} \frac{1}{M^{3 / 2}} \mathrm{e}^{-\frac{x^{2}}{2 W}} .
$$

Therefore the probability $h(W, l) \mathrm{d} W$ that configuration $\mathrm{C}_{1}$ falls on an attractor of period $l$ and of weight larger than $W$ but less that $W+d W$ is given by

$$
\begin{aligned}
h(W, l) \mathrm{d} W & =R_{n}(l) M \mathrm{~d} W \\
& =\frac{1}{\sqrt{2 \pi M W(1-W)}} \mathrm{e}^{-\frac{x^{2}}{2 W}} .
\end{aligned}
$$

\section{Distribution $\pi(Y)$.}

In addition to the weights $W$ of valleys, there is another quantity $Y$ which has been considered in the mean field theory of spin glasses. ( $Y$ is the length of the plateau of the function $q(x))$. It is defined by

$$
Y=Y_{2}=\sum_{s} W_{s}^{2} \text {. }
$$

In this section we are going to obtain the probability distribution of $Y$. The first moments are easy to obtain, from the knowledge of $g(W), g\left(W_{1}, W_{2}\right)$ and $g\left(W_{1}, W_{2}, W_{3}\right)$ :

$$
\bar{Y}=\int_{0}^{1} \mathrm{~d} W W g(W)=\frac{2}{3}
$$

$$
\bar{Y}^{2}=\int_{0}^{1} \mathrm{~d} W_{1} \int_{0}^{1} \mathrm{~d} W_{2} W_{1} W_{2} g\left(W_{1}, W_{2}\right)=\frac{52}{105}
$$

$$
\begin{aligned}
\bar{Y}^{3}=\int_{0}^{1} \mathrm{~d} W_{1} \int_{0}^{1} \mathrm{~d} W_{2} \int_{0}^{1} \mathrm{~d} W_{3} W_{1} W_{2} W_{3} \times \\
\times g\left(W_{1}, W_{2}, W_{3}\right)=\frac{376}{945} .
\end{aligned}
$$


One can in principle continue and obtain like that all the moments of $Y$. There is however an easier way which gives a recursion relation for the moments $\bar{Y}^{n}$.

If we consider a configuration $\mathrm{C}_{1}$, we know that it has a probability $g(W)$ of falling on an attractor of weight $W$. We can therefore consider phase space as composed of the basin of the attractor of $\mathrm{C}_{1}$ which contains $M W$ points, and of the remaining $N(1-W)$ points. The restriction $\tilde{T}$ of the map $T$ to these $N(1-W)$ remaining points is again a random map and therefore should have the same statistical properties as the map $T$. Therefore one can write

$$
Y=W^{2}+(1-W)^{2} \tilde{Y}
$$

where $Y$ corresponds to the map $T$ and $\tilde{Y}$ corresponds to the map $\tilde{T}$. One should notice that $Y$ and $W$ are correlated whereas $\tilde{Y}$ and $W$ are not. From (47), it is easy to compute all the moments $\overline{Y^{n}}$ using the fact that $Y$ and $\tilde{Y}$ have the same moments. Taking the $n$-th power of (47) one gets

$$
\begin{aligned}
\overline{Y^{n}}=\sum_{p=0}^{n} & \frac{n !}{p !(\tilde{n}-p) !} \overline{W^{2(n-p)}(1-W)^{2 p}} \overline{Y^{p}}= \\
= & \sum_{p=0}^{n} \frac{n !}{p !(n-p) !} \overline{Y^{p}} \\
& \times \int_{0}^{1} \mathrm{~d} W g(W) W^{2(n-p)}(1-W)^{2 p}
\end{aligned}
$$

One can then compute $\overline{Y^{n}}$ knowing $\overline{Y^{p}}$ for $1 \leqq$ $p \leqq n-1$ :

$$
\begin{aligned}
\bar{Y}^{n}= & \frac{1}{4} \sum_{p=0}^{n-1} \overline{Y^{p}} \times \\
& \times \frac{\Gamma\left(2 p+\frac{1}{2}\right) \Gamma(2 n-2 p+1) \Gamma(n)}{\Gamma(p+1) \Gamma(n-p+1) \Gamma\left(2 n+\frac{1}{2}\right)} .
\end{aligned}
$$

It is easy to check that for $N=1,2,3$ one recovers (46).

Once all the moments $\overline{Y^{n}}$ of $Y$ are known, the distribution $\pi(Y)$ of $Y$ is known (since the support of $\pi(Y)$ is the interval $[0,1])$. It is however not easy to obtain the explicit expression of $\pi(Y)$ from the $\overline{Y^{n}}$.

To obtain a picture of $\pi(Y)$, we use a stochastic method based on formula (47). We just calculate a random sequence $\left\{y_{n}\right\}$ where $y_{0}$ is randomly chosen between 0 and 1 , where the recursion relation which gives the $y_{n}$ is

$$
y_{n+1}=W_{n}^{2}+\left(1-W_{n}\right)^{2} y_{n}
$$

and where the $W_{n}$ are chosen randomly according to the probability distribution

$$
g\left(W_{n}\right)=1 / 2\left(1-W_{n}\right)^{-1 / 2} .
$$

Then we know that the $y_{n}$ which are generated by this method are distributed according to $\pi(Y)$. In figure 1 we draw the histogram of the $y_{n}$ obtained by iterating the recursion relation (50) $10^{7}$ times.

We see that the shape of $\pi(Y)$ is again very similar to what has been obtained in the mean field theory of spin glasses [18]. We see in figure 1 that $\pi(Y)$ diverges at $Y=1$ and has singularities at $Y=1 / 2$ and $Y=1 / 3$.

Using the same argument which led to (47) and (50), one can compute other statistical properties of the random map model. For example one can obtain easily the probability $\tilde{\pi}\left(W_{\max }\right)$ that the largest basin of attraction has a weight $W_{\max }$. This is easily achieved by drawing the histogram of the $y_{n}$ which are obtained by the recursion

$$
y_{n+1}=\max \left(W_{n},\left(1-W_{n}\right) y_{n}\right)
$$

where again the $W_{n}$ are distributed according to $g\left(W_{n}\right)$ (see Fig. 2).

Lastly, it is easy to generalize the result (49) to obtain the more general moments $\overline{\left(Y_{\delta}\right)^{n}}$ where $Y_{\delta}$ is defined by

$$
Y_{\delta}=\sum_{s} W_{s}^{\delta} .
$$

For $Y_{\delta}$ there is of course a recursion relation analogous to (47) :

$$
Y_{\delta}=W^{\delta}+(1-W)^{\delta} \tilde{Y}_{\delta}
$$

where again $Y_{\delta}$ and $\tilde{Y}_{\delta}$ have the same distribution. Then one finds that the moments $\overline{\left(Y_{\delta}\right)^{n}}$ are given by

$$
\begin{aligned}
\overline{Y_{\delta}^{n}}= & \frac{1}{2 \delta} \sum_{p=0}^{n-1} \overline{Y_{\delta}^{p}} \times \\
& \times \frac{\Gamma\left(p \delta+\frac{1}{2}\right) \Gamma((n-p) \delta+1) \Gamma(n)}{\Gamma(p+1) \Gamma(n-p+1) \Gamma\left(n \delta+\frac{1}{2}\right)} .
\end{aligned}
$$

Of course for $\delta=2$, one recovers (49).

\section{Conclusion.}

In the present work, we have described several statistical properties of the multivalley structure of the random map model. Our main conclusion is that these properties are very similar although not identical to those known in the mean field theory of spin glasses. This rises the question whether a replica approach could be developed for the Kauffman model and whether this replica approach would lead to a replica symmetry breaking. 


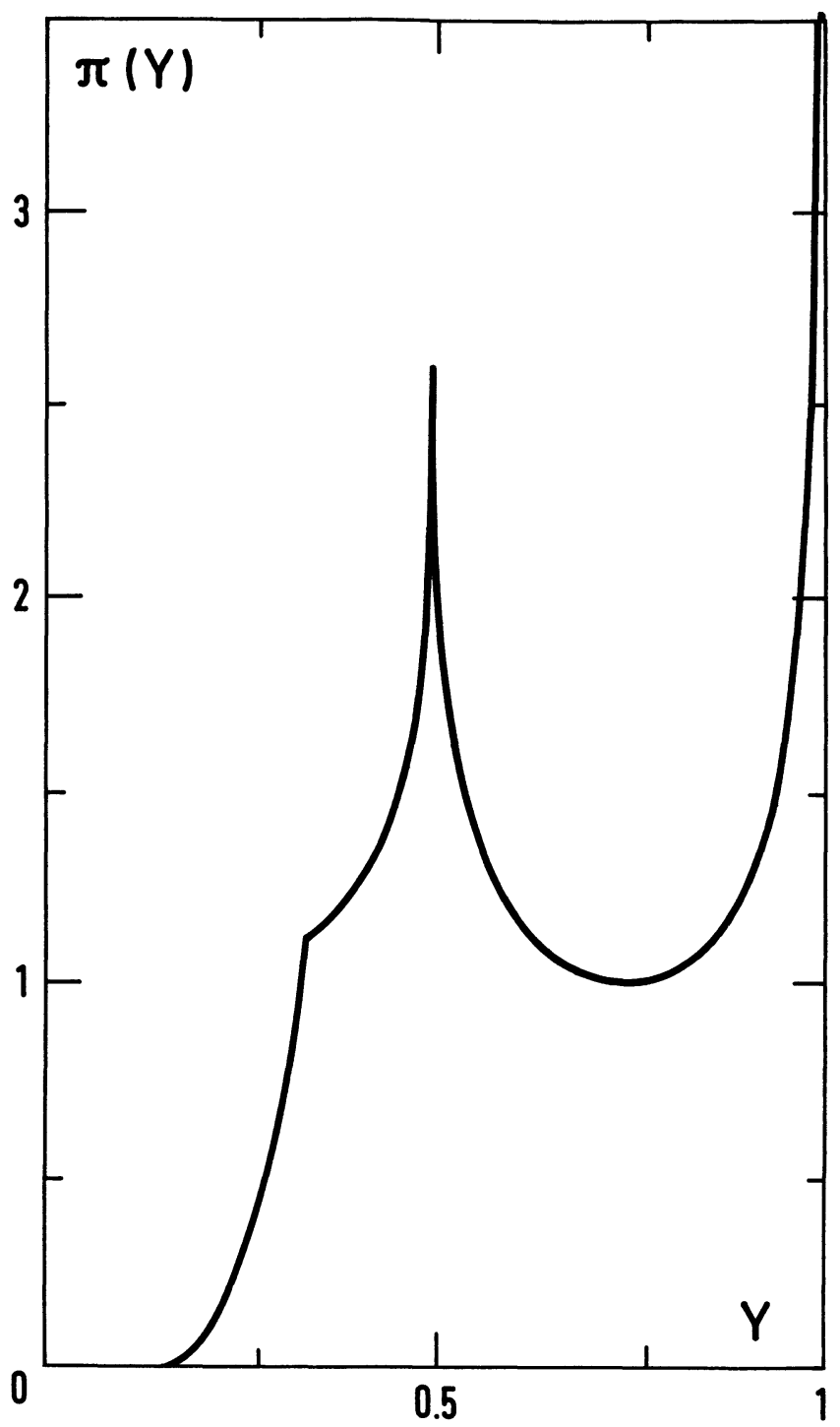

Fig. 1. - Probability distribution $\pi(Y)$ obtained by iterating $10^{7}$ times the recursion (46). $Y$ is defined by equation (41).

The random map model is related to the Kauffman model like the random energy model [28] is related to the Sherington-Kirkpatrick model. In spin glasses, correlations between energies lead, within the replica approach, to the ultrametric structure of valleys. It would be interesting to see whether the Kauffman model for finite $K$ presents a similar structure.

\section{Acknowledgments.}

B. D. would like to thank the Institute of Theoretical Physics, UCSB, where part ot this work was done. This research was supported in part by the U.S. National Science Foundation under Grant No. PHY82-17853 supplemented by funds from the U.S. National Aeronautics and Space Administration.

\section{Appendix.}

In this appendix we show that equation (31) is equivalent to equations (33) and (34).

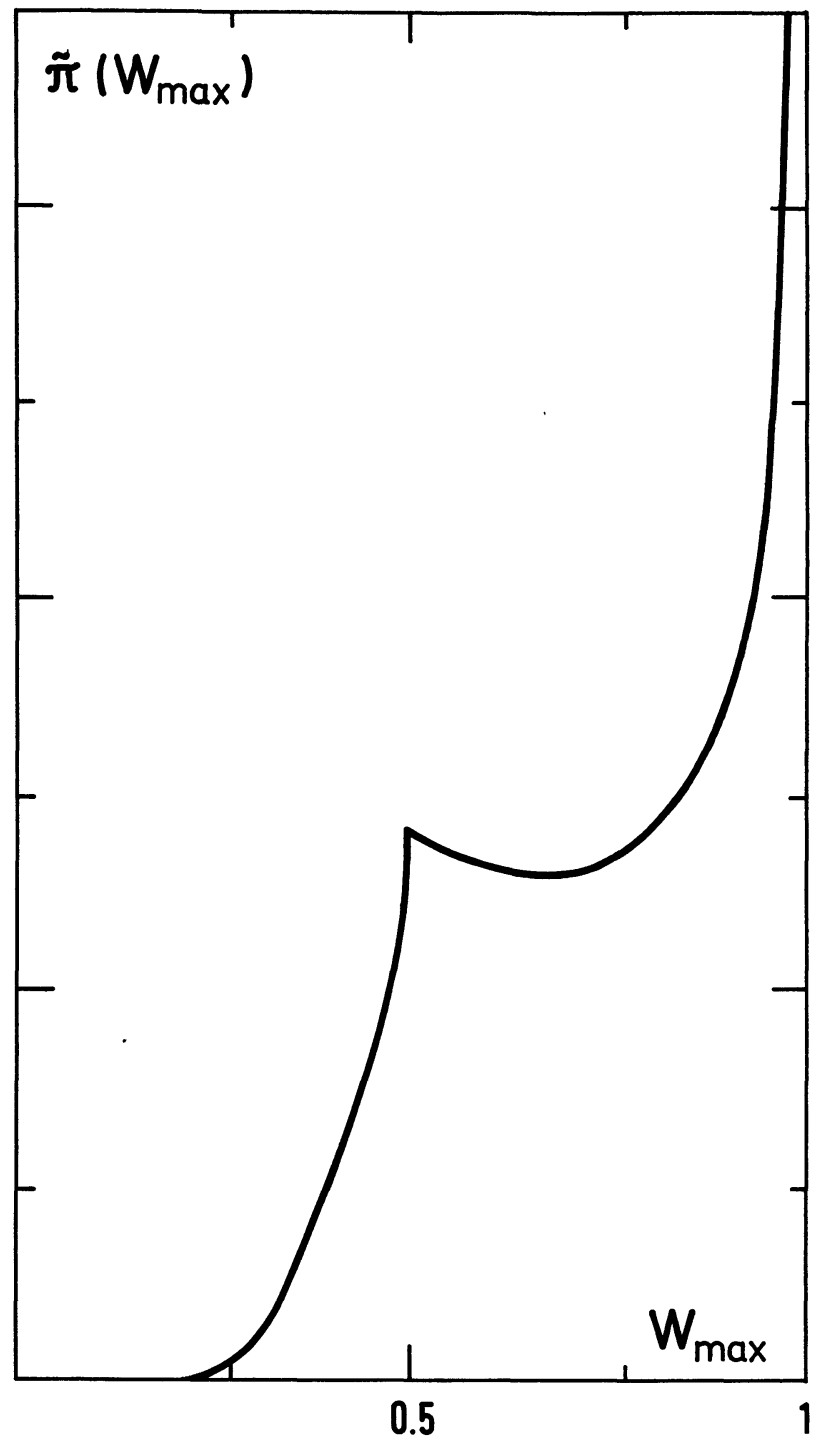

Fig. 2. - Probability distribution $\tilde{\pi}\left(W_{\max }\right)$ of the weight $W_{\max }$ of the largest valley.

We have to show that $\overline{Z_{n}(l)}=A$ given by (31)

$$
A=\frac{1}{\sqrt{M}} \frac{1}{(n-1) !} \frac{1}{2^{n-1}} \int_{x}^{\infty} \mathrm{d} y \mathrm{e}^{-y^{2} / 2}\left(y^{2}-x^{2}\right)^{n-1}
$$

is equivalent to $\overline{Z_{n}(l)}=B$ given by (33) and (34)

$$
B=\int_{0}^{1} \mathrm{~d} W W^{n-1} \frac{1}{\sqrt{2 \pi M}} \frac{1}{\sqrt{W(1-W)}} \mathrm{e}^{-\frac{x^{2}}{2 W}}
$$

Let us first transform (A.1) :

If

$$
\begin{aligned}
& x^{2} / 2=a \\
& y^{2} / 2=t
\end{aligned}
$$


then (A.1) gives

$A=\frac{1}{\sqrt{M}} \frac{1}{\Gamma(n)} \frac{1}{\sqrt{2}} \int_{a}^{\infty} \mathrm{d} t t^{-1 / 2}(t-a)^{n-1} \mathrm{e}^{-t}$

and if one writes

$$
W=\frac{x^{2}}{2 t}
$$

then (A.2) becomes

$$
B=\frac{a^{n-\frac{1}{2}}}{\sqrt{2 \pi M}} \int_{a}^{\infty} \mathrm{d} t t^{-n}(t-a)^{-1 / 2} \mathrm{e}^{-t} .
$$

So we have to prove that (A.5) and (A.7) are the same. One can rewrite (A.7) as :

$$
\begin{aligned}
B=\frac{1}{\Gamma(n)} \frac{a^{n-\frac{1}{2}}}{\sqrt{2 \pi M}} \int_{a}^{\infty} \mathrm{d} t t^{-n}(t-a)^{-\frac{1}{2}} \times \\
\times \mathrm{e}^{-t} \int_{0}^{\infty} \mathrm{d} t_{1} t_{1}^{n-1} \mathrm{e}^{-t_{1}}
\end{aligned}
$$

where we have just used the definition of $\Gamma(n)$.

Then in (A.8) we make the following change of variable

$$
t_{1}=t u
$$

One then gets

$$
\begin{aligned}
& B=\frac{1}{\Gamma(n)} \frac{a^{n-\frac{1}{2}}}{\sqrt{2 \pi M}} \int_{a}^{\infty} \mathrm{d} t \times \\
& \times \int_{0}^{\infty} \mathrm{d} u(t-a)^{-1 / 2} u^{n-1} \mathrm{e}^{-t(1+u)} .
\end{aligned}
$$

If one writes now

$$
t=a+\frac{x}{1+u}
$$

(A.10) becomes

$$
\begin{aligned}
B & =\frac{1}{\Gamma(n)} \frac{a^{n-\frac{1}{2}}}{\sqrt{2 \pi M}} \int_{0}^{\infty} \mathrm{d} x \times \\
& \times \int_{0}^{\infty} \mathrm{d} u x^{-\frac{1}{2}}(1+u)^{-\frac{1}{2}} u^{n-1} \mathrm{e}^{-x-a(1+u)} .
\end{aligned}
$$

The integral over $x$ is easy and one gets

$B=\frac{1}{\Gamma(n)} \frac{a^{n-\frac{1}{2}}}{\sqrt{2 M}} \int_{a}^{\infty} \mathrm{d} u u^{n-1}(1+u)^{-\frac{1}{2}} \mathrm{e}^{-a(1+u)}$.

A last change of variable

$$
t=a(1+u)
$$

leads to (A.5) and therefore proves that $\mathrm{A}=\mathrm{B}$.

\section{References}

[1] Bienenstock, M. Y., Fogelman Soulié, F. and WeIsBuCH, G., 1986. Disordered Systems and Biological Organization (Springer Verlag, Heidelberg) and references therein.

[2] Kauffman, S. A., J. Theor. Biol. 22 (1969) 437.

[3] Kauffman, S. A., Physica D 10 (1984) 145.

[4] Atlan, H., Fogelman Soulié, F., Salomon, J. and WeIsbuch, G., Cybern. Syst. 12 (1981) 103.

[5] Fogelman Soulié, F. (1985), Thesis, Grenoble University.

[6] Derrida, B. and Pomeau, Y., Europhys. Lett. 1 (1986) 45.

[7] Derrida, B. and Weisbuch, G., J. Physique 47 (1986) 1297.

[8] DerridA, B. and Stauffer, D., Europhys. Lett. 2 (1986) 739.

[9] Weisbuch, G. and Stauffer, D., J. Physique 48 (1987) 11.

[10] Hilhorst, H. J. and NiJMEIJER, M., preprint 1986.

[11] Little, W. and Shaw, G., Math. Biosci. 39 (1978) 281.

[12] Personnaz, L., Guyon, I. and Dreyfus, G., J. Physique Lett. 46 (1985) L-359.

[13] KANTER, I. and SOMPOLINSKY, H., preprint 1986.

[14] Gardner, E., Derrida, B. and Mottishaw, P., preprint 1986.
[15] Derrida, B. and FlyvbJerg, H., J. Phys. A 19 (1986) L1001.

[16] Sherington, D. and KirkPatrick, S., Phys. Rev. Lett. 32 (1975) 1792.

[17] Mezard, M., Parisi, G., Sourlas, N., Toulouse, G. and Virasoro, M., Phys. Rev. Lett. 52 (1984) 1146.

[18] Mezard, M., Parisi, G., Sourlas, N., Toulouse, G. and Virasoro, M., J. Physique 45 (1984) 843.

[19] Mezard, M., Parisi, G. and Virasoro, M., J. Physique Lett. 46 (1985) 217.

[20] Derrida, B. and Toulouse, G., J. Physique Lett. 46 (1985) 223.

[21] Harris, B., Ann. Math. Stat. 31 (1960) 1045.

[22] Metropolis, N. and Ulam, S., Am. Math. Monthly 60 (1953) 252.

[23] Kruskal, M. D., Am. Math. Monthly 61 (1954) 392.

[24] Katz, L., Ann. Math. Stat. 26 (1955) 512.

[25] Rubin, H. and Sitgreaves, R., Probability distributions related to random transformations on a finite set, report No. 19A, Stanford University 1954 , probably unpublished.

[26] LeVY, Y. E., Phys. Lett. 88A (1982) 1.

[27] COSTE, J. and HENON, M., p. 361 in reference [1].

[28] Derrida, B., Phys. Rev. Lett. 45 (1980) 79. 